\title{
Criterios para publicar casos clínicos
}

\author{
JULIO PERTUZÉ R.*
}

\section{Criteria for publishing case reports}

\section{Introducción}

La publicación de casos clínicos constituye una de las formas más antiguas de comunicación científica médica. Ya Osler los describió como observaciones registradas cuidadosamente para producir educación y ser fuente de investigaciones valiosas ${ }^{1}$. En la actualidad se considera que su contribución al conocimiento científico es relativamente escasa, por cuanto pueden contener prejuicios y carecen de control para evitar los efectos del azar. Por ello no constituyen una evidencia confiable para la toma de decisiones en la práctica clínica, para lo cual tienen la mayor jerarquía los estudios prospectivos, aleatorios, controlados ${ }^{2,3}$. Por ejemplo, el aporte de un caso que comunica un determinado efecto adverso a drogas es muy inferior al de un trabajo multicéntrico que describe un número significativo de pacientes con efectos adversos semejantes, o al de una comunicación que establece la frecuencia con que se presenta dicho efecto en un elevado número de pacientes con ese tratamiento.

Sin embargo, pese a que la mayoría de los comités editoriales de publicaciones científicas coinciden en que la comunicación de casos clínicos contribuye escasamente al conocimiento, ellos valoran su publicación como una forma de comunicación entre clínicos con propósitos educativos $^{4-6}$. Del mismo modo que el análisis de casos clínicos constituye una técnica fundamental en la enseñanza médica de pregrado, su publicación es una fuente de enseñanza amena que facilita el aprendizaje continuo de los médicos. Así como las anécdotas ilustran una exposición, captan la atención, ayudan a recordarla y favorecen su comprensión, de igual modo la lectura de un caso clínico permite recordar mensajes, incrementar el abanico de diagnósticos diferenciales o motivar la búsqueda de más información. Por ello existe acuerdo en que estas publicaciones constituyen una importante herramien- ta docente. Por otra parte, su redacción en un lenguaje cercano al de la práctica clínica diaria puede hacerlas más atractivas y por lo tanto más didácticas para un clínico con menos experiencia en lectura crítica de la literatura médica.

Aun cuando la publicación de casos o series clínicas sea el nivel más bajo de evidencia, pueden aportar conocimiento al describir un caso o evento centinela, es decir, la primera evidencia de una situación, diagnóstica, terapéutica o pronóstica, inusual o inesperada. Esta puede constituir una advertencia que ponga en alerta ante situaciones semejantes, estimule la generación de estudios de epidemiología clínica metodológicamente apropiados, o de otras investigaciones relevantes para responder las preguntas que puedan haber surgido del caso. Quizás el ejemplo más ilustrativo al respecto sea la comunicación de los casos de neumonía por $P$. carinii en hombres homosexuales previamente sanos ${ }^{7}$.

En relación a la publicación de series de casos clínicos, estas suelen tener limitaciones; la obtención de historias clínicas completas en forma retrospectiva es difícil y en muchos casos no se logra un seguimiento adecuado que permita asegurar que las conclusiones no hubieran sido diferentes al incluir los casos faltantes. Estas consideraciones determinan que algunas publicaciones prioricen la presentación de casos clínicos individuales por sobre la publicación de series de casos retrospectivos ${ }^{2}$.

Por último, la publicación de casos clínicos puede servir de incentivo a académicos jóvenes que trabajan en el medio asistencial. La satisfacción de ver sus casos publicados puede motivarlos para generar proyectos de investigación en áreas relacionadas ${ }^{8}$.

\section{Características que justifican su publicación}

Dado que el objetivo de la publicación de casos clínicos es básicamente educacional, los autores deben tener presente el mensaje que quie-

* Departamento de Enfermedades Respiratorias, Pontificia Universidad Católica de Chile. 
ren comunicar y si este conocimiento será útil a los lectores. El caso debe interesar a quienes trabajan en el área relacionada y a quienes suelen enfrentar ese tipo de pacientes. Por lo mismo, la condición no debe ser tan inusual que los lectores de esa publicación raramente vayan a enfrentar un caso semejante. Es conveniente revisar la literatura médica para investigar si el motivo por el cual se pretende publicar ya ha sido descrito a raíz de casos similares, cuántas veces y bajo qué circunstancias. La revisión bibliográfica, además, puede aportar información sobre los exámenes o procedimientos específicos que se requieren para confirmar el diagnóstico para incluirlos en la descripción.

Los casos aptos para publicación no deben ser necesariamente raros, pueden ser condiciones con formas de presentación, manejo o evolución inhabitual. Suelen caer en las categorías descritas en la Tabla 1.

Algunas publicaciones exigen que el caso sea único, que ilustre sólo un aspecto clínico educativo, y que describa tratamientos nuevos, inusuales o innovadores no descritos previamen$\mathrm{te}^{4}$. No obstante, la publicación de casos ya comunicados puede ser útil si contribuye a poner en evidencia la real magnitud del problema, motivar la búsqueda de explicaciones fisiopatológicas u originar investigaciones prospectivas. Asimismo, aun cuando ya se hayan descrito en forma aislada situaciones similares, puede ser conveniente su difusión en un determinado medio local.

\section{Estructura de la publicación}

Para que la presentación cumpla sus propósitos, su estructura debe incluir el motivo por el

\section{Tabla 1. Casos aptos para ser publicados como casos clínicos}

- Condición o enfermedad nueva
- Condición rara, infrecuente y poco comunicada
- Presentación inusual de una enfermedad común
- Asociación inesperada entre síntomas o signos infre-
cuentes
- Impacto de una enfermedad en la evolución de otra
- Evolución inusual o evento inesperado en el curso
de una observación o tratamiento
- Impacto del tratamiento de una condición en otra
- Complicaciones inesperadas de procedimientos o tra-
tamientos (efectos colaterales no descritos)
- Tratamientos o procedimientos diagnósticos nuevos
y "únicos"

* Adaptado de Chekvarajah et $\mathrm{al}^{6}$. cual se presenta el caso, un breve resumen de los eventos que lo caracterizan, una revisión selectiva de la bibliografía que ponga el caso en su contexto y concluir ya sea sugiriendo al lector una lección concreta de diagnóstico o manejo o destacando cómo la observación descrita aporta o desafía conocimientos 9 . Junto con estar enfocada al público para el cual el tema o mensaje es de interés, debe redactarse de acuerdo a las normas de la revista a la que se enviará para publicación.

Como toda comunicación científica, su estructura debe incluir:

- Título: Que sea claro y atractivo, incluyendo palabras claves relevantes que permitan el rescate electrónico de la información

- Resumen: Con breve descripción de la situación clínica señalando por qué el caso es importante o destacable, especificando el mensaje educativo que motiva su comunicación

- Introducción: Que ponga el caso clínico en contexto en términos de frecuencia y gravedad de los síntomas/signos/enfermedad y su relevancia para el público al cual está destinado. Esta sección puede omitirse si estos aspectos han sido incluidos en el resumen.

- Descripción del caso: Con la necesaria fundamentación que le otorgue credibilidad. Narra el proceso diagnóstico en forma tal que queda claro que el diagnóstico es el correcto y, cuando proceda, destacar que se consideraron todas las posibles opciones terapéuticas justificando la opción elegida.

- Discusión y comentarios: Enfatizando por qué el caso es destacable y explicar o clarificar los aspectos discutibles. Debe incluir las lecciones que pueden aprenderse del caso que se comenta, precisando el mensaje educacional, sea práctica a imitar, errores a evitar o implicancias diagnósticas o pronósticas que pueden cuestionarse a la luz de esta experiencia.

- Conclusiones y recomendaciones: Destacar en forma sucinta el mensaje que debe quedar de su lectura, las recomendaciones para el manejo de pacientes similares o las líneas de investigación que podrían originarse a propósito de este caso. La conclusión ha de ser cauta por cuanto no se puede recomendar tratamientos o extrapolar conclusiones a raíz de sólo un caso en particular.

- Referencias: Es importante limitar las referencias bibliográficas a las pertinentes para avalar la discusión; destacar un concepto clínico, recordar lo infrecuente o importante de una condición, o apoyar la necesidad de cambiar determinadas prácticas clínicas. 
No es conveniente efectuar una extensa revisión de la literatura médica, propia de los artículos de revisión, que tienen otra estructura, y generalmente son encargados por los comités editoriales a personas con vasta experiencia en el tema.

Asimismo, se debe tomar precauciones para evitar que la descripción del caso permita la identificación del paciente y, si ello no es posible, obtener consentimiento informado de éste o sus familiares antes de la publicación ${ }^{10}$.

Para cumplir su papel didáctico, el caso clínico debe redactarse en forma coherente con un principio, desarrollo y conclusión que refleje el razonamiento diagnóstico y terapéutico de quien lo presenta, aun cuando no siga el esquema tradicional de la historia clínica descrita en textos de semiología ${ }^{11}$. Cuando sea necesario, la descripción debe incluir precozmente elementos de la anamnesis remota, $\mathrm{u}$ otros antecedentes relevantes que faciliten la comprensión del caso $^{12}$. Así por ejemplo ante un paciente que consulta por disnea y tos, cuya anamnesis remota incluye el diagnóstico de infección recurrente del tracto urinario e ingesta de nitrofurantoina, la incorporación precoz de esta información, facilita su comprensión. Una redacción amena favorece el objetivo docente.

\section{Criterios para evaluar la publicación}

El caso clínico debe ser juzgado por su importancia, claridad y lo práctico de su mensaje educacional. Sobre la base de lo anterior, propongo evaluar los casos considerando los siguientes aspectos:

1. La novedad, originalidad o poco conocimiento del tema, por lo que su publicación constituye un aporte al describir una situación nueva, aumentar la casuística de una enfermedad poco habitual o por constituir una forma de presentación poco habitual de una enfermedad conocida (Tabla 1).

2. Redacción y facilidad de lectura. Al respecto evaluar si:

a) El título es apropiado y permite la recuperación de la información.

b) El resumen destaca la importancia del caso y su mensaje.

c) La descripción del diagnóstico y opciones terapéuticas está adecuadamente fundamentada.

d) Calidad de la discusión: es pertinente al caso, incluye un análisis de los aspectos discutibles, y avala el mensaje que motiva la presentación. e) Concluye con un mensaje educativo claro, preciso y acorde con la información presentada.

f) El caso clínico está redactado globalmente en forma amena y fácil de leer.

3. Pertinencia y calidad de la revisión bibliográfica.

4. Ausencia de reparos éticos

5. Utilidad de la publicación.

Considerar si el mensaje es de interés para los lectores de la revista y su difusión constituye un aporte para el medio local. Es decir, cuánto aporta al lector el haber conocido la publicación, y en qué manera el conocimiento de este caso puede influir en el enfrentamiento o manejo de problemas similares.

Probablemente el mensaje más importante para el autor que presenta un caso clínico y para el revisor antes de recomendar su publicación, es que se planteen muy honestamente estas 3 preguntas: ¿Se habrían interesado en leer este caso clínico si lo hubieran encontrado al hojear la revista? ¿Qué lecciones aprendieron de él? ¿Qué mensaje les quedó?

\section{Bibliografías}

1.- CUSHING H W. The life of Sir William Osler. Oxford University Press Inc. Oxford 1926.

2.- EDITORIAL: Case reports, case series and systematic reviews. QJ Med 2002; 95: 197-8.

3.- MATTHEWS J R. Quantification and the Quess for Medical Certainty. Princeton, New Jersey: Princeton Univ. Press 1995.

4.- KAALID S K, THOMPSON P J. A proposal for writing and appraising case reports. Br J Obstet Gynaecol 2002; 109; 849-51.

5.- VANDENBROUKE J P. In defence of case reports and case series. Ann Intern Med 2001; 134: 330-4.

6.- CHELVARAJAH R, BYCORFT J. Writing and publishing case reports, the road to success. Acta Neurochir (Wien) 2004; 146: 313-6.

7.- GOTTLIEB M S, SCHROFF R, SCHANKER H M, WEISMAN J D, FAN $\mathrm{P}$ T, WOLF $\mathrm{R}$ A, et al. Pneumocystis carinii pneumonia and mucosal candidiasis in previously healthy homosexual men: evidence of a new acquired cellular immunodeficiency. N Engl J Med 1981; 305: 1425-31.

8.- PATOTE S, WHITEHALL J. Publishing case reportsa powerful tool for academia motivation. Postgrad Med J 2000; 76: 597.

9.- MORGAN P P. Why case reports? Can Med Assoc J 1985; 133: 353.

10.- LEVINE S B, STAGNO S J. Informed consent for case reports: the ethical dilemma of right to privacy versus pedagogical freedom. J Psycother Pract Res 2001; 10: 193-201.

11.- GOIC A, CHAMORRO G, REYES H. Semiología Médica, $2^{\circ}$ edición. Publicaciones Técnicas Mediterráneo 1999; pp 325-9.

12.- KOPPLIN P A. The storied case report. Can Med Assoc J 2004; 171: 569-70. 